\title{
ZERO DIVISORS AND NILPOTENT ELEMENTS IN POWER SERIES RINGS
}

\author{
DAVID E. FIELDS ${ }^{1}$
}

ABSTRACT. It is well known that a polynomial $f(X)$ over a commutative ring $R$ with identity is nilpotent if and only if each coefficient of $f(X)$ is nilpotent; and that $f(X)$ is a zero divisor in $R[X]$ if and only if $f(X)$ is annihilated by a nonzero element of $R$. This paper considers the problem of determining when a power series $g(X)$ over $R$ is either nilpotent or a zero divisor in $R[[X]]$. If $R$ is Noetherian, then $g(X)$ is nilpotent if and only if each coefficient of $g(X)$ is nilpotent; and $g(X)$ is a zero divisor in $R[[X]]$ if and only if $g(X)$ is annihilated by a nonzero element of $R$. If $R$ has positive characteristic, then $g(X)$ is nilpotent if and only if each coefficient of $g(X)$ is nilpotent and there is an upper bound on the orders of nilpotency of the coefficients of $g(X)$. Examples illustrate, however, that in general $g(X)$ need not be nilpotent if there is an upper bound on the orders of nilpotency of the coefficients of $g(X)$, and that $g(X)$ may be a zero divisor in $R[[X]]$ while $g(X)$ has a unit coefficient.

1. Introduction. It is well known that a polynomial $f(X)$ over a commutative ring $R$ with identity is nilpotent if and only if each coefficient of $f(X)$ is nilpotent. In [1], McCoy establishes that a polynomial $f(X)$ is a zero divisor in $R[X]$ if and only if there is a nonzero element $r$ of $R$ with $r f(X)=0$. In this paper, we consider the problem of determining when a power series $g(X)$ over $R$ is either nilpotent or a zero divisor in $R[[X]]$. We prove (Corollary 1 ) that if $R$ is Noetherian, then $g(X)$ is nilpotent if and only if each coefficient of $g(X)$ is nilpotent. And if $R$ is Noetherian, then $g(X)$ is a zero divisor of $R[[X]]$ if and only if $g(X)$ is annihilated by some nonzero element of $R$ (Theorem 5). We establish (Theorem 1) that if $R$ has positive characteristic, then $g(X)$ is nilpotent if and only if each coefficient of $g(X)$ is nilpotent and there is an upper bound on the orders of nilpotency of the coefficients of $g(X)$. We show by means of examples, however, that, in general, $g(X)$ need not be nilpotent if there is an upper bound on the orders of nilpotency of the coefficients

Presented to the Society, April 18, 1970; received by the editors October 23, 1969 and, in revised form, February 26, 1970.

AMS 1970 subject classifications. Primary 13A15, 13 B99.

Key words and phrases. Formal power series, zero divisors, nilpotent elements.

1 This paper is part of the author's dissertation, written under the direction of Professor Robert Gilmer of Florida State University. 
of $g(X)$, and that $g(X)$ may be a zero divisor while $g(X)$ has a unit coefficient.

Throughout this paper, $R$ denotes a commutative ring with identity; $\omega$ is the set of natural numbers; $\omega_{0}$ is the set of nonnegative integers; $Z$ is the set of integers; and $Q$ is the set of rational numbers. If $f(X)=\sum_{i=0}^{\infty} f_{i} X^{i} \in R[[X]]$, we denote by $A_{f}$ the ideal of $R$ generated by the coefficients of $f(X): A_{f}=\left\{f_{0}, f_{1}, f_{2}, \cdots\right\} R$. If $A$ is an ideal of $R$, we let $A[[X]]=\left\{f(X)=\sum_{i=0}^{\infty} f_{i} X^{i}: f_{i} \in A\right.$ for each $\left.i \in \omega_{0}\right\}$ and we define $A \cdot R[[X]]$ to be the ideal of $R[[X]]$ which is generated by $A$. Then $A \cdot R[[X]]=\left\{f(X): A_{f} \subseteq B\right.$ for some finitely generated ideal $B$ of $R$ with $B \subseteq A\}$. It is clear that $A \cdot R[[X]] \subseteq A[[X]]$; equality holds if and only if each countably generated ideal of $R$ contained in $A$ is contained in a finitely generated ideal contained in $A$. In particular, if $V$ is a valuation ring containing an ideal $A$ which is countably generated but not finitely generated, then $A \cdot V[[X]]$ $C A[[X]]$. Finally, we note that if $A$ is an ideal of $R$, then $R[[X]] / A[[X]] \simeq(R / A)[[X]]$; hence $A[[X]]$ is a prime ideal of $R[[X]]$ if and only if $A$ is a prime ideal of $R$.

2. Nilpotent elements. Let $f(X)=\sum_{i=0}^{\infty} f_{i} X^{i} \in R[[X]]$ and let $n \in \omega_{0}$; we define $f^{(n)}=\sum_{i=0}^{n} f_{i} X^{i}$. Then $f^{(n)}$ is zero or a polynomial of degree at most $n$.

LEMMA 1. Let $R$ be a commutative ring with identity having characteristic a positive prime $p$, and let $f(X)=\sum_{i=0}^{\infty} f_{i} X^{i} \in R[[X]]$. The following conditions are equivalent:

(a) $f(X)$ is nilpotent.

(b) There is a natural number $m$ such that $\left(f_{i}\right)^{m}=0$ for each $i \in \omega_{0}$.

(c) There is a natural number $m$ such that $\left(f^{(k)}\right)^{m}=0$ for each $k \in \omega_{0}$.

Proof. (a) $\leftrightarrow$ (b): This follows immediately from the fact that for each natural number $n,(f(X))^{p^{n}}=\sum_{i=0}^{\infty}\left(f_{i}\right)^{p^{n}} X^{i p^{n}}$.

(b) $\leftrightarrow$ (c): This is clear since for each natural number $n$ and for each nonnegative integer $k,\left(f^{(k)}\right)^{p^{n}}=\sum_{i=0}^{k}\left(f_{i}\right)^{p^{n}} X^{i p^{n}}$.

THEOREM 1. Let $R$ be a commutative ring with identity having positive characteristic $n=p_{1}^{e_{1}} p_{2}^{e_{2}} \cdots p_{i}^{e_{t}}$ and let $f(X)=\sum_{i=0}^{\infty} f_{i} X^{i} \in R[[X]]$. The following conditions are equivalent:

(a) $f(X)$ is nilpotent.

(b) There is a natural number $m$ such that $\left(f_{i}\right)^{m}=0$ for each $i \in \omega_{0}$.

(c) There is a natural number $m$ such that $\left(f^{(k)}\right)^{m}=0$ for each $k \in \omega_{0}$.

Proof. We let $\phi_{j}: R[[X]] \rightarrow R[[X]] / p_{j} R[[X]]$ be the natural homomorphism for $1 \leqq j \leqq t$. We note that for $1 \leqq j \leqq t, R / p_{j} R$ has characteristic $p_{j}$. 
(a) $\rightarrow$ (b): If $f(X)$ is nilpotent, then for $1 \leqq j \leqq t, \phi_{j}(f(X))$ is nilpotent in $R[[X]] / p_{j} R[[X]] \simeq\left(R / p_{j} R\right)[[X]]$. By Lemma 1 , there is, for $1 \leqq j \leqq t$, a natural number $m_{j}$ satisfying: For $i \in \omega_{0}, 0=\left(\phi_{j}\left(f_{i}\right)\right)^{m_{i}}$ $=\phi_{j}\left(\left(f_{i}\right)^{m_{j}}\right)$; that is, $\left(f_{i}\right)^{m_{j}} \in p_{j} R$ for each $i \in \omega_{0}$.

Let $m=m_{1} e_{1}+m_{2} e_{2}+\cdots+m_{t} e_{t}$. Then for each $i \in \omega_{0}$,

$$
\left(f_{i}\right)^{m}=\left(f_{i}^{m_{1}}\right)^{e_{1}}\left(f_{i}^{m_{2}}\right)^{e_{2}} \cdots\left(f_{i}^{m_{i}}\right)^{e_{t}} \in\left(p_{1} R\right)^{e_{1}}\left(p_{2} R\right)^{e_{2}} \cdots\left(p_{t} R\right)^{e_{t}}=(0) .
$$

Hence (b) holds.

(b) $\rightarrow$ (a): We assume that there is a natural number $m$ satisfying: $\left(f_{i}\right)^{m}=0$ for each $i \in \omega_{0}$. Then for each $j, 1 \leqq j \leqq t, \phi_{j}\left(\left(f_{i}\right)^{m}\right)=\left(\phi_{j}\left(f_{i}\right)\right)^{m}$ $=0$ for each $i \in \omega_{0}$. By Lemma 1 , there is for each $j, 1 \leqq j \leqq t$, a natural number $m_{j}$ satisfying

$$
\left[\phi_{j}(f(X))\right]^{m_{j}}=\phi_{j}\left((f(X))^{m_{j}}\right)=0 ; \quad \text { that is, }(f(X))^{m_{j}} \in p_{j} R[[X]] .
$$

Let $m=m_{1} e_{1}+m_{2} e_{2}+\cdots+m_{t} e_{t}$; then

$$
\begin{aligned}
(f(X))^{m} & =\left[(f(X))^{m_{1}}\right]_{e_{1}}\left[(f(X))^{m_{2}}\right]_{e_{2}} \cdots\left[(f(X))^{m_{t}}\right]^{e_{t}} \\
& \in\left(p_{1} R[[X]]\right)^{e_{1}}\left(p_{2} R[[X]]\right)^{e_{2}} \cdots\left(p_{t} R[[X]]\right)^{e_{t}}=(0) .
\end{aligned}
$$

Hence $f(X)$ is nilpotent.

The proof that (b) $\leftrightarrow$ (c) is analogous to the proof that (a) $\leftrightarrow$ (b); hence it will be omitted.

THEOREM 2. Let $R$ be a commutative ring with identity and let $f(X)$ $=\sum_{i=0}^{\infty} f_{i} X^{i} \in R[[X]]$. We consider the following conditions:

(a) The ideal $A_{f}$ is nilpotent.

(b) There is a natural number $m$ which salisfies: $\left[A_{f^{(k)}}\right]^{m}=(0)$ for each $k \in \omega_{0}$.

(c) There is a natural number $m$ which satisfies: $\left[f^{(k)}\right]^{m}=0$ for each $k \in \omega_{0}$.

(d) There is a natural number $m$ which satisfies: $\left(f_{i}\right)^{m}=0$ for each $i \in \omega_{0}$.

(e) There is a natural number $m$ which satisfies: $\left[f^{(k)}\right]^{m} \in\left(X^{k+1}\right)$ $\cdot R[[X]]$ for each $k \in \omega_{0}$.

(f) $f(X)$ is nilpotent.

We have the implications $(\mathrm{a}) \leftrightarrow(\mathrm{b}) \rightarrow(\mathrm{c}) \rightarrow(\mathrm{e}) \leftrightarrow(\mathrm{f})$ and $(\mathrm{c}) \rightarrow(\mathrm{d})$.

Proof. (a) $\rightarrow$ (b): For each $k \in \omega_{0}, A_{f}^{(k)} \subseteq A_{f}$; hence if $\left(A_{f}\right)^{m}=(0)$, then $\left[A_{f}{ }^{(k)}\right]^{m}=(0)$ for each $k \in \omega_{0}$.

(b) $\rightarrow\left(\right.$ a): Let $m$ be a natural number which satisfies: $\left[A_{f^{(k)}}\right]^{m}=(0)$ for each $k \in \omega_{0}$. Let $a \in\left(A_{f}\right)^{m}$; then for some $i \in \omega_{0}, a \in\left[A_{f^{(i)}}\right]^{m}=(0)$. Thus $\left(A_{f}\right)^{m}=(0)$.

(b) $\rightarrow$ (c): Obvious. 
$\left(\right.$ c) $\rightarrow\left(\right.$ d) : Let $m$ be a natural number which satisfies: $\left[f^{(k)}\right]^{m}=0$ for each $k \in \omega_{0}$. Then for each $i \in \omega_{0},\left(f_{i}\right)^{m}=\left(\left[f^{(i)}\right]^{m}\right)_{i m}=0$.

$(\mathrm{c}) \rightarrow(\mathrm{e}):$ Clear.

(e) $\rightarrow$ (f): We first observe that if $i \leqq k$, then for each $m \in \omega$,

$$
\left([f(X)]^{m}\right)_{i}=\sum_{\substack{e_{1}+e_{2}+\cdots+e_{g}=m \\ r_{1} e_{1}+r_{2} e_{2}+\cdots+r_{s} e_{s}=i}}\left(n_{r_{1} r_{2}} \cdots r_{s} f_{r_{1}}^{e_{1}} f_{r_{2}}^{e_{2}} \cdots f_{r_{s}}^{e_{g}}\right)
$$

where each $n_{r_{1} r_{2}} \ldots r_{s} \in \omega$, implying that $\left([f(X)]^{m}\right)_{i}=\left(\left[f^{(k)}\right]^{m}\right)_{i}$. For if $r_{1} e_{1}+r_{2} e_{2}+\cdots+r_{8} e_{s}=i$ with each $r_{i} \in \omega_{0}$ and each $e_{i} \in \omega$, then $r_{8} \leqq i \leqq k$. Thus only the coefficients $f_{0}, f_{1}, \cdots, f_{t}$, where $t \leqq k$, occur in the calculation of $\left([f(X)]^{m}\right)_{i}$, whereby we obtain the above equality.

Assuming (e), let $m$ be a natural number which satisfies: $\left[f^{(k)}\right]^{m}$ $\in\left(X^{k+1}\right) R[[X]]$ for each $k \in \omega_{0}$. Then for each $j \in \omega_{0}, \quad\left([f(X)]^{m}\right)_{j}$ $=\left(\left[f^{(j)}\right]^{m}\right)_{j}=0$ since $\left[f^{(j)}\right]^{m} \in\left(X^{j+1}\right) R[[X]]$. Hence $[f(X)]^{m}=0$ and $f(X)$ is nilpotent.

$(\mathrm{f}) \rightarrow(\mathrm{e}):$ We assume that $[f(X)]^{m}=0$; then whenever $i \leqq k, 0$ $=\left([f(X)]^{m}\right)_{i}=\left(\left[f^{(k)}\right]^{m}\right)_{i}$. Thus $\left[f^{(k)}\right]^{m} \in\left(X^{k+1}\right) R[[X]]$ for each $k \in \omega_{0}$ and (e) holds.

COROLlaRY 1. Let $R$ be a commutative ring with identity and let $f(X)=\sum_{i=0}^{\infty} f_{i} X^{i} \in R[[X]]$. If $A_{f}$ is a finitely generated ideal of $R$, then the following conditions are equivalent:

(a) The ideal $A_{f}$ is nilpotent.

(c) There is a natural number $m$ which satisfies: $\left[f^{(k)}\right]^{m}=0$ for each $k \in \omega_{0}$.

(d) There is a natural number $m$ which satisfies: $\left(f_{i}\right)^{m}=0$ for each $i \in \omega_{0}$.

(f) $f(X)$ is nilpotent.

(g) Each coefficient of $f(X)$ is nilpotent.

Proof. In Theorem 2, we established the implications (c) $\rightarrow$ (d) and $(\mathrm{a}) \rightarrow(\mathrm{c}) \rightarrow(\mathrm{f})$. That $(\mathrm{d}) \rightarrow(\mathrm{g})$ is clear. Hence it suffices to prove that $(\mathrm{f}) \rightarrow(\mathrm{g})$ and that $(\mathrm{g}) \rightarrow(\mathrm{a})$.

$(\mathrm{g}) \rightarrow(\mathrm{a}):$ If $(\mathrm{g})$ holds, then each element of $A_{f}$ is nilpotent. Since $A_{f}$ is finitely generated, $A_{f}$ is nilpotent.

$(\mathrm{f}) \rightarrow(\mathrm{g})$ : Let $\left\{P_{\alpha}\right\}$ be the collection of prime ideals of $R$ and let $N$ be the ideal of nilpotent elements of $R$; then $N=\bigcap_{\alpha} P_{\alpha}$. For each $\alpha$, $P_{\alpha}[[X]]$ is a prime ideal of $R[[X]]$. Since $f(X)$ is nilpotent, $f(X)$ $\in P_{\alpha}[[X]]$ for each $\alpha$. Hence $f(X) \in \bigcap_{\alpha} P_{\alpha}[[X]]=\left(\bigcap_{\alpha} P_{\alpha}\right)[[X]]$ $=N[[X]]$; that is, each coefficient of $f(X)$ is nilpotent. 
We now give examples which show that in Theorem $2,(\mathrm{c}) \nrightarrow(\mathrm{a})$ and (d) $\nrightarrow$ (f).

ExAMPLE 1 . Let $S=Z /(p)$ where $p$ is a positive prime; let $\left\{X_{i}\right\}_{i \in \omega_{0}}$ be a countable collection of indeterminates over $S$; and let

$$
\begin{aligned}
R= & S\left[X_{0}, X_{1}, \cdots, X_{t}, \cdots\right] /\left\{X_{0}^{p}, X_{1}^{p}, \cdots, X_{t}^{p}, \cdots\right\} \\
& \cdot S\left[X_{0}, X_{1}, \cdots, X_{t}, \cdots\right] .
\end{aligned}
$$

Let $f_{i}=\bar{X}_{i}$ and let $f(X)=\sum_{i=0}^{\infty} f_{i} X^{i} \in R[[X]]$. Then for each $k \in \omega_{0}$, $\left[f^{(k)}\right]^{p}=0$. But for each natural number $n, f_{0} f_{1} \cdots f_{n-1} \in\left(A_{f}\right)^{n}$ and $f_{0} f_{1} \cdots f_{n-1} \neq 0$. Thus $A_{f}$ is not nilpotent. We conclude that (c) $\nrightarrow$ (a).

EXAMPLE 2. Let $n \in \omega, n \geqq 2$, and let

$$
\begin{aligned}
R= & Q\left[X_{0}, X_{1}, \cdots, X_{t}, \cdots\right] /\left\{X_{0}^{n}, X_{1}^{n}, \cdots, X_{t}^{n}, \cdots\right\} \\
& \cdot Q\left[X_{0}, X_{1}, \cdots, X_{t}, \cdots\right] .
\end{aligned}
$$

Let $f_{i}=\bar{X}_{i}$ and let $f(X)=\sum_{i=0}^{\infty} f_{i} X^{i} \in R[[X]]$. It is clear that $\left(f_{i}\right)^{n}=0$ for each $i \in \omega_{0}$; hence $f(X)$ satisfies $(\mathrm{d})$.

We assume that $f(X)$ is nilpotent: $[f(X)]^{m}=0$. Then $f_{0}^{m}$ $=\left([f(X)]^{m}\right)_{0}=0$ so $m \geqq n$. Let $k_{1}$ be the smallest integer $t$ for which $f_{0}^{n}$ does not occur in every summand used in computing $\left([f(X)]^{m}\right)_{t}$. Then $0=\left([f(X)]^{m}\right)_{k_{1}}=a f_{0}^{n-1} f_{1}^{m-(n-1)}$ plus other terms, each having $f_{0}^{n}$ as a factor, where $a \in \omega$. Hence $0=\left([f(X)]^{m}\right)_{k_{1}}=a f_{0}^{n-1} f_{1}^{m-(n-1)}$, implying that $m-(n-1) \geqq n$.

Let $k_{2}$ be the smallest integer $t$ for which some summand used in computing $\left([f(X)]^{m}\right)_{t}$ has neither $f_{0}^{n}$ nor $f_{1}^{n}$ as a factor. Then 0 $=\left([f(X)]^{m}\right)_{k_{2}}=b f_{0}^{n-1} f_{1}^{n-1} f_{2}^{m-2(n-1)}$ plus other terms, each having either $f_{0}^{n}$ or $f_{1}^{n}$ as a factor, where $b \in \omega$. Hence $0=\left([f(X)]^{m}\right)_{k_{2}}=b f_{0}^{n-1} f_{1}^{n-1}$ $\cdot f_{2}^{m-2(n-1)}$, implying that $m-2(n-1) \geqq n$.

We can prove inductively by this process that for each $k \in \omega$, $m-k(n-1) \geqq n$; that is, $m \geqq n+k(n-1)$. This contradicts our assumption that $m \in \omega$, showing that $f(X)$ is not nilpotent. Hence (d) $\nrightarrow$ (f).

\section{Zero divisors.}

LEMMA 2. Let $R$ be a commutative ring with identity and let $f(X)$ $=\sum_{i=0}^{\infty} f_{i} X^{i} \in R[[X]]$. If for some natural number $t, f_{t}$ is regular in $R$ while $f_{i}$ is nilpotent for $0 \leqq i \leqq t-1$, then $f(X)$ is regular in $R[[X]]$.

Proof. We let $g(X)=\sum_{i=0}^{t-1} f_{i} X^{i}$ and $h(X)=\sum_{i=t}^{\infty} f_{i} X^{i}$; then $f(X)=g(X)+h(X)$. (We let $g(X)=0$ if $t=0$.) Since $g(X)=0$ or $g(X)$ 
is a polynomial of which each coefficient is nilpotent, $g(X)$ is nilpotent.

Let $T$ denote the total quotient ring of $R$ and let $S=T[[X]]_{M}$ where $M=\left\{X^{i}\right\}_{i=1}^{\infty}$. Then in $S$, we can write $h(X)=X^{t} h^{\prime}(X)$ where $h^{\prime}(X)=\sum_{i=0}^{\infty} f_{i+t} X^{i}$; thus $h(X)$ and $h^{\prime}(X)$ are associates in $S$. Since $f_{t}=\left(h^{\prime}(X)\right)_{0}$ is regular in $R, f_{t}$ is a unit of $T$, implying that $h^{\prime}(X)$ is a unit in $T[[X]]$, hence also in $S$. Since $h(X)$ and $h^{\prime}(X)$ are associates in $S, h(X)$ is a unit in $S$. Hence in $S, f(X)=g(X)+h(X)$ where $g(X)$ is nilpotent and $h(X)$ is a unit, implying that $f(X)$ is a unit, hence is regular, in $S$ [2, Exercise 18, p. 9]. Thus $f(X)$ is regular in $R[[X]]$.

THEOREM 3. Let $R$ be a commutative ring with identity in which each zero divisor is nilpotent, and let $f(X)=\sum_{i=0}^{\infty} f_{i} X^{i} \in R[[X]]$. If some $f_{i}$ is regular in $R$, then $f(X)$ is regular in $R[[X]]$.

Proof. This is an immediate consequence of Lemma 2, letting $t$ be the smallest integer $k$ for which $f_{k}$ is regular in $R$.

COROLlaRY 2. Let $R$ be a commutative ring with identity in which each zero divisor is nilpotent. If the ideal $N$ of nilpotent elements of $R$ is nilpotent, then in $R[[X]]$ each zero divisor is nilpotent.

Proof. Let $f(X)=\sum_{i=0}^{\infty} f_{i} X^{i} \in R[[X]]$ and assume that $f(X)$ is not nilpotent. Then $A_{f}$ is not nilpotent so $A_{f} \Phi N$; that is, not every coefficient of $f(X)$ is nilpotent. By assumption, $f(X)$ has a regular coefficient. By Theorem $3, f(X)$ is regular in $R[[X]]$.

We observe that Corollary 2 can be restated as follows:

CoROLlary 3. Let $R$ be a commutative ring with identity in which (0) is $N$-primary. If $N$ is nilpotent, then (0) is a primary ideal of $R[[X]]$.

We immediately have the following:

CoROLlaRy 4. Let $R$ be a commutative ring with identity and let $Q$ be a P-primary ideal of $R$. If $Q \supseteq P^{k}$ for some $k \in \omega$, then $Q[[X]]$ is a $P[[X]]$-primary ideal of $R[[X]]$.

Proof. Since $R[[X]] / Q[[X]] \simeq(R / Q)[[X]]$, it follows from Corollary 3 that $Q[[X]]$ is a primary ideal of $R[[X]]$. Also, $P^{k} \subseteq Q$ so that $(P[[X]])^{k} \subseteq P^{k}[[X]] \subseteq Q[[X]]$; hence $P[[X]]=\sqrt{ }\left[(P[[X]])^{k}\right]$ $\subseteq \sqrt{ } Q[[X]]$. And clearly $\sqrt{ } Q[[X]] \subseteq P[[X]]$. Hence $\sqrt{ } Q[[X]]$ $=P[[X]]$ and $Q[[X]]$ is $P[[X]]$-primary.

THEOREM 4. Let $R$ be a Noetherian ring with identity in which $(0)=Q_{1} \cap Q_{2} \cap \cdots \cap Q_{n}$ is a shortest primary representation, with $\sqrt{ } Q_{i}=P_{i}$. Then in $R[[X]], \quad(0)=Q_{1}[[X]] \cap Q_{2}[[X]]$ 
$\cap \ldots \cap Q_{n}[[X]]$ is a shortest primary representation with $\sqrt{ } Q_{i}[[X]]$ $=P_{i}[[X]]$.

Proof. $Q_{1}[[X]] \cap \cdots \cap Q_{n}[[X]]=\left(Q_{1} \cap \cdots \cap Q_{n}\right)[[X]]=(0)$. Further, Corollary 4 asserts that each $Q_{i}[[X]]$ is $P_{i}[[X]]$-primary. It is straightforward to verify that this primary representation of (0) in $R[[X]]$ is, in fact, irredundant.

THEOREM 5. Let $R$ be a Noetherian ring with identity in which $(0)=Q_{1} \cap Q_{2} \cap \cdots \cap Q_{n}$ is a shortest primary representation of (0) with $\sqrt{ } Q_{i}=P_{i}, 1 \leqq i \leqq n$. Then for $f(X)=\sum_{i=0}^{\infty} f_{i} X^{i} \in R[[X]]$, these conditions are equivalent:

(a) $f(X)$ is a zero divisor in $R[[X]]$.

(b) $f(X) \in P_{i}[[X]]$ for some $i, 1 \leqq i \leqq n$.

(c) There is a nonzero element $r$ of $R$ which satisfies $r f(X)=0$.

Proof. (a) $\rightarrow$ (b): This is an immediate consequence of Theorem 4 and [3, Corollary 3, p. 214].

(b) $\rightarrow$ (c): Assuming that $f(X) \in P_{i}[[X]]$, this implies that $A_{f} \subseteq P_{i}$. Thus $(0): A_{f} \neq(0)$ by $\left[3\right.$, Corollary 1, p. 214]. Let $r \in(0): A_{f}, r \neq 0$; then clearly $r \in R$ and $r \neq 0$ while $r f(X)=0$.

(c) $\rightarrow$ (a): Obvious.

We conclude with an example which shows that Theorem 5 fails when $R$ is not Noetherian.

EXAMPLE $3 .^{2}$ Let $S$ be a commutative ring with identity; let $\left\{Y, X_{0}, X_{1}, X_{2}, \cdots, X_{t}, \cdots\right\}$ be a set of indeterminates over $S$; and let

$$
R=S\left[Y,\left\{X_{i}\right\}_{i=0}^{\infty}\right] /\left(X_{0} Y,\left\{X_{i}-X_{i+1} Y\right\}_{i=0}^{\infty}\right) .
$$

Let $y=\bar{Y}$ and let $f(X)=y-X$. Then $f(X)$ has a unit coefficient, so certainly $r f(X) \neq 0$ for each nonzero element $r$ of $R$. However, letting $x_{i}=\bar{X}_{i}$ and $g(X)=\sum_{i=0}^{\infty} x_{i} X^{i}$, we see that $f(X) \cdot g(X)=0$ while $g(X) \neq 0$.

\section{REFERENCES}

1. Neil H. McCoy, Remarks on divisors of zero, Amer. Math. Monthly 49 (1942), 286-295. MR 3, 262.

2. - The theory of rings, Macmillan, New York, 1964. MR $32 \# 5680$.

3. O. Zariski and P. Samuel, Commutative algebra. Vol. I, The University Series in Higher Math., Van Nostrand, Princeton, N. J., 1958. MR 19, 833.

Stetson University, Deland, Florida 32720

Florida State University, Tallahassee, Florida 32306

2 Example 3 was pointed out to the author by Professor Gilmer. 